\title{
Editorial: Supplemental Material or Joint Submission?
}

As described in https:https://journals.aps.org/authors/supplemental-materials-journals, Supplemental Material (SM) is information that will be useful to a subset of readers, but is not essential to the comprehension of the main results of the published article. The Physical Review journals archive SM to provide permanent access to information such as multimedia files, tables of raw or analyzed data, parameters used in or produced by calculations, computer code, sample preparation, derivations of equations, etc. SM cannot be crucial to a reader's understanding - the paper must be self-contained and convincing without it.

Importantly, SM should not be used to avoid a length limit, so a short paper accompanied by a lengthy supplement may not be appropriate. For a submittal to Physical Review Letters (PRL), authors may consider an option made possible by our close-knit family of journals: the simultaneous submission of a longer version to one of the more specialized Physical Review journals. That longer version should provide a substantially increased understanding of the subject; otherwise, the use of SM may be better.

The submission of an expanded version of a PRL to a topical Physical Review journal is an established practice that provides readers with easier access to important additional information. If authors simultaneously submit a Letter to PRL and a regular paper to one of the Physical Review journals, the two manuscripts are then reviewed coherently, typically by the same reviewers. If both papers receive favorable reviews, we aim to publish them at the same time, unless this leads to undue delay for one of them. We also ensure that the paper and the Letter cite each other.

Submitting a joint publication couldn't be easier. Simply alert the editors by mentioning the companion papers in your cover letter.

Published 14 June 2017

Pierre Meystre

Editor in Chief

American Physical Society

DOI: 10.1103/PhysRevLett.118.240001 